\title{
Chemical Composition of the Essential Oil of Mastic Gum and their Antibacterial Activity Against Drug-Resistant Helicobacter pylori
}

\author{
Tomofumi Miyamoto • Tadayoshi Okimoto • \\ Michihiko Kuwano
}

Received: 4 June 2014/ Accepted: 2 July 2014/Published online: 19 July 2014

(C) The Author(s) 2014. This article is published with open access at Springerlink.com

\begin{abstract}
Mastic gum is derived from the tree named Pistacia lentiscus that is grown only in Island Hios of Greek. Since Mastic was first reported to kill Helicobacter pylori (H. pylori) in 1998, there has been no further study to elucidate which component of mastic specifically shows the antimicrobial activity against $H$. pylori. In this study, we examined which component of mastic gum was responsible for anti-H. pylori activity. We prepared the essential oil of mastic gum and identified 20 constituents by GC-MS analysis. Ten standard components were assayed for anti-H. pylori activity, and it clarified that $\alpha$-terpineol and $(E)$-methyl isoeugenol showed the anti-H. pylori activity against four different $H$. pylori strains that were established from patients with gastritis, gastric ulcer and gastric cancer. These components could be useful to overcome the drug-resistance H. pylori growth in stomach.
\end{abstract}

Keywords Mastic gum $\cdot$ Pistacia lentiscus $\cdot$ Anti-Helicobacter pylori $\cdot \alpha$-terpineol $\cdot($ E)-methyl isoeugenol

\section{Introduction}

Helicobacter pylori (H. pylori), gram-negative bacterium, induces chronic gastric infection of one-half of the world population. Infection with $H$. pylori is often associated with a viable proportion of duodenal ulcer, gastric ulcer, and gastric carcinoma [1, 2]. During chronic infection process of $H$. pylori for decades, the bacterium infection initially induces chronic gastritis, and progresses to atrophic

T. Miyamoto $(\square)$

Department of Natural Products Chemistry, Graduate School of Pharmaceutical Sciences, Kyushu University, Fukuoka, Japan

e-mail: miyamoto@phar.kyushu-u.ac.jp

T. Okimoto

Department of Gastroenterology, Oita University Faculty of Medicine, Oita, Japan

e-mail: okimoto@oita-u.ac.jp

M. Kuwano

Laboratory of Molecular Cancer Biology, Graduate School of Pharmaceutical Sciences, Kyushu University, Fukuoka, Japan e-mail: kuwano@phar.kyushu-u.ac.jp gastritis and metaplasia and then to cancer [3]. Intestinaltype gastric cancer shows a multi-step carcinogenic process, from atrophic gastritis to intestinal metaplasia to dysplasia. In Japan, Fukase et al. [4] reported that gastric cancer was inhibited by $H$. pylori eradication in postendoscopic gastric mucosal resection of gastric cancer in a multi-center randomized control trial. As an approach for prevention of gastric cancer, $H$. pylori infection has been eradicated by combined treatment with several antimiciobial agents plus proton pump inhibitors. However, this approach does not always provide satisfactory benefits for whole population because of the high cost of therapeutic agents and the emergence of antibiotic resistance [5]. A new approach for anti-H. pylori vaccines has seen recently developed, and some of these vaccines have had some success in the eradicative animal models, and efforts to prove these vaccines efficaciens are underway in human vaccine trials [6].

Further development of potent antimicrobial drugs is expected to improve the therapeutic and preventive effects against pathogenesis by $H$. pylori. Zaidi et al. [7] have isolated bactericidal constituents from the plant named 
Table 1 Chemical composition of the essential oil of mastic gum

\begin{tabular}{|c|c|c|c|c|c|c|c|}
\hline No. & Compound & $t_{\mathrm{R}}(\min )$. & $\%$ & No. & Compound & $t_{\mathrm{R}}(\min )$. & $\%$ \\
\hline 1 & $\alpha$-pinene & 5.4 & 82.26 & 11 & $\alpha$-terpineol & 13.8 & 0.77 \\
\hline 2 & $\beta$-pinene & 6.4 & 2.96 & 12 & $p$-cymene-8-ol & 14.1 & 0.54 \\
\hline 3 & $\beta$-myrcene & 6.7 & 1.92 & 13 & myrtenal & 14.2 & 0.29 \\
\hline 4 & $p$-cymene & 7.8 & 0.41 & 14 & verbenone & 14.7 & 1.50 \\
\hline 5 & limonene & 7.9 & 0.84 & 15 & $(E)$-carveol & 15.0 & 0.23 \\
\hline 6 & linalool & 10.4 & 1.50 & 16 & 2-undecanone & 17.9 & 0.16 \\
\hline 7 & camphenal & 11.4 & 0.31 & 17 & $\beta$-caryophyllene & 22.7 & 0.73 \\
\hline 8 & pinocarvenal & 12.0 & 1.25 & 18 & $\alpha$-caryophyllene & 24.0 & 0.09 \\
\hline 9 & verbenol & 12.2 & 0.71 & 19 & $(E)$-Me isoeugenol & 25.6 & 0.07 \\
\hline 10 & myrcenol & 13.2 & 0.43 & 20 & caryphyllene oxide & 28.5 & 0.14 \\
\hline
\end{tabular}

Mallotus philippinenesis that are effective against clarithromycin-and metronidazole-resistant strains of Japanese and Pakistani $H$. pylori. On the other hand, mastic gum, which is a resin secreted from the stem of Pistacia lentiscus, was found to be effective against $H$. pylori [8]. In our present study, we examined which component of the essential oil prepared from mastic gum could inhibit the growth of $H$. pylori. The bactericidal activity of the mastic components will be discussed in association with their drug resistance reversal effects.

\section{Results and Discussion}

\subsection{Chemical Composition of the Essential Oil of Mastic Gum}

GC-MS analysis of the essential oil of mastic gum led to the identification of the components, which are listed in Table 1. A typical GC-MS chromatogram of the essential oil of mastic gum is illustrated in Fig. 1. The identification of the components was based on comparison of their mass spectra with those of NIST and Wiley libraries, as well as on comparison of their retention times $[9,10]$ and of the standard components analyzed. The major constituent of the essential oil was $\alpha$-pinene (Peak 1;82.26\%), and totally 20 components were identified from the essential oil of mastic gum.

\subsection{Antibacterial Activity of Mastic Components}

We next examined which component inhibits the growth of H. pylori. Commercially available 10 compounds were tested for antibacterial activities against $H$. pylori clinical strains. The results were summarized in Table 2. Surprisingly, some of them showed antibacterial activity againt clarithromycin (CAM)- and/or metronidazole (MNZ)resistant strains. Figure 2 shows most potent anti-pyloritic activity of $(E)$-methyl isoeugenol and $\alpha$-terpineol not only against drug sensitive strains (\#09-292) but also against drug resistant strains (\#09-87, \#09-224 and \#09-243). These 10 compounds also showed antibacterial activity against three different strains (E. coli, S. aureus, B. subtilis) [9].

The antibacterial activity of the mastic gum was first reported about two decades ago [11]. In 2007, Paraschos et al. reported that the acidic fraction of mastic gum showed the potent bactericidal activity against $H$. pylori clinical strains and the most active compound was isomasticadienolic acid [12]. In our screening analysis of the essential oil of mastic gum, we identified 20 chemical compositions, and an $(E)$-methyl isoeugenol was found to show the potent antibacterial activity against four $H$. pylori strains including CAM- and MNZ-resistant strain. Another compound, $\alpha$-terpineol also showed antibacterial activity, but its effect was less than that of $(E)$-methyl isoeugenol. In the previous report [9], verbenone, $\alpha$-terpineol, and linalool showed higher antibacterial activity than all other components, and $(E)$-methyl isoeugenol showed moderate activity against E. coli, S. aureus, B. subtillis. (E)-methyl isoeugenol and $\alpha$-terpineol are the trace components of the essential oil. However, in our present study, these components first showed much higher antibacterial activity against $H$. pylori when compared with the same dose of the essential oil itself.

H. pylori infection is known to be involved in gastric and duodenum ulcer, gastritis and metaplasia, gastric cancer and MALT lymphoma [13-17]. H. pylori infection can be eradicated by combined treatment with several antimicrobial agents plus proton pump inhibitors. Fukase et al. have reported that the eradication of $H$. pylori reduces the incidence of gastric cancer [4]. In Japan, combined treatment with proton pump inhibitor (PPI), amoxicillin (AMPC) and CAM have been applied as first line eradication therapy, and MNZ is replaced CAM as second line eradication therapy [18]. However, the subsequent increase in bacterial resistance to CAM in Japan 


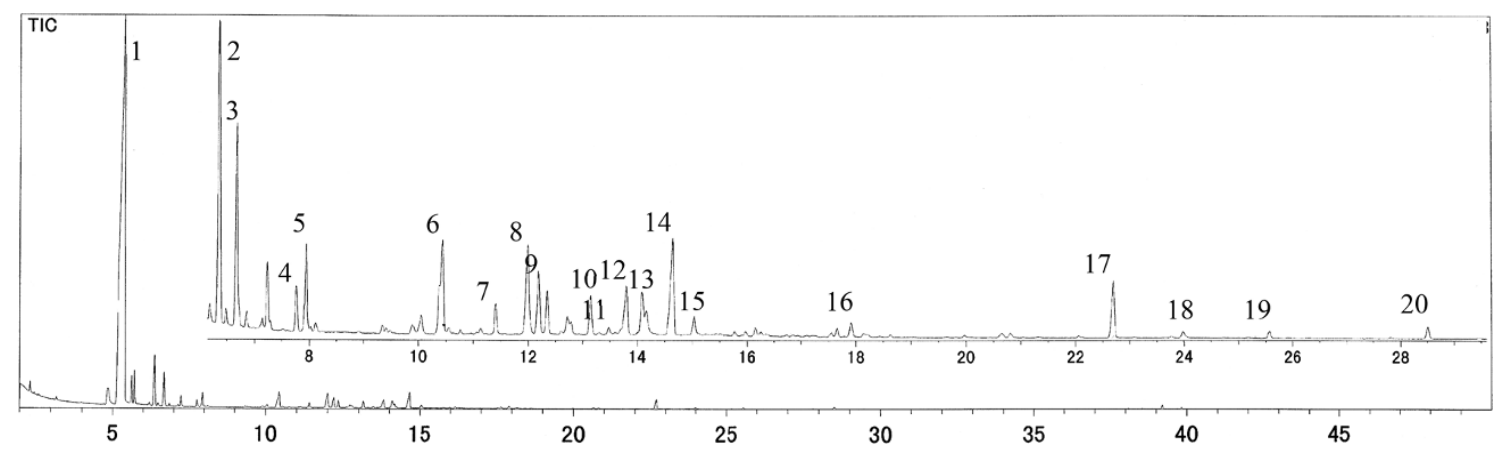

Fig. 1 GC-MS chromatogram of the essential oil of mastic gum

caused a decline in the eradication rate of first line therapy [19]. Our present study demonstrated antibacterial effects of $(E)$-methyl isoeugenol and $\alpha$-terpineol against CAMand MNZ-resistant $H$. pylori strains (Table 2). These compounds derived from the mastic gum could be further useful for eradication of $H$. pylori including drug-resistant H. pyroli.

\section{Experimental Section}

\subsection{Material and Chemicals}

Mastic gum was purchased from Sunsho Pharmaceutical Co. Ltd. (Fujinomiya, Japan). Standard compounds, $\alpha$ pinene, $\beta$-pinene, $\beta$-caryophyllene, terpineol were purchased from Sigma Aldrich Japan (Tokyo, Japan), $\beta$-myrcene, $p$-cymene, linallol, anethole, verbenone from Wako Pure Chemical Industries, Ltd. (Osaka, Japan), limonene and $(E)$-methyl isoeugenol from Tokyo Chemical Industry Co. Ltd. (Tokyo, Japan).

\subsection{Preparation of the Essential Oil of Mastic Gum}

The essential oil was prepared according to the manufactural protocol in the Japanese Pharmacopoeia. Mastic gums $(30 \mathrm{~g})$ in a $300 \mathrm{~mL}$ round bottom flask was set to a prescribed distillation apparatus and refluxing with $300 \mathrm{~mL}$ of distilled water for $5 \mathrm{~h}$ to yield $0.9 \mathrm{~mL}$ of essential oil (731.2 mg, $2.4 \%$ yield).

\subsection{Chemical Composition of the Essential Oil of Mastic Gum}

The GC-MS analysis of the essential oil was undertaken using a Shimadzu QP-5050A GC-MS system (Kyoto, Japan), operating in electron ionization (EI) mode with an ionization energy of $70 \mathrm{eV}$. The instrument was equipped with an INERTCAP-5MS/SIL capillary column $(30 \mathrm{~m}$,
Table 2 Antibacterial activity (zones of inhibition, $\mathrm{mm}$ ) of the selected compounds of the essential oil of mastic gum

\begin{tabular}{|c|c|c|c|c|c|}
\hline Compounds & $\mu \mathrm{g} / \mathrm{disk}^{\mathrm{a}}$ & $\# 09-87^{\mathrm{b}}$ & $\# 09-224^{\mathrm{b}}$ & $\# 09-243^{b}$ & $\# 09-292^{\mathrm{b}}$ \\
\hline \multirow[t]{2}{*}{$\alpha$-pinene } & 10 & - & - & - & - \\
\hline & 100 & 10 & 12 & - & 13 \\
\hline \multirow[t]{2}{*}{$\beta$-pinene } & 10 & - & - & - & - \\
\hline & 100 & - & - & - & - \\
\hline \multirow[t]{2}{*}{$\beta$-myrcene } & 10 & - & - & 10 & - \\
\hline & 100 & - & - & - & - \\
\hline \multirow[t]{2}{*}{$p$-cymene } & 10 & - & - & - & - \\
\hline & 100 & 11 & 11 & - & - \\
\hline \multirow[t]{2}{*}{ limonene } & 10 & - & - & - & - \\
\hline & 100 & 11 & 11 & 11 & 12 \\
\hline \multirow[t]{2}{*}{ linalool } & 10 & - & - & - & - \\
\hline & 100 & 15 & 14 & 14 & 14 \\
\hline \multirow[t]{2}{*}{$\alpha$-terpineol } & 10 & - & - & - & - \\
\hline & 100 & 17 & 20 & 18 & 22 \\
\hline \multirow[t]{2}{*}{ verbenone } & 10 & - & - & - & - \\
\hline & 100 & 11 & 11 & - & 11 \\
\hline \multirow[t]{2}{*}{$\beta$-caryophyllene } & 10 & - & - & - & - \\
\hline & 100 & 12 & 10 & - & - \\
\hline \multirow{2}{*}{$\begin{array}{l}\text { (E)-methyl } \\
\text { isoeugenol }\end{array}$} & 10 & - & 10 & - & 15 \\
\hline & 100 & 25 & 23 & 35 & 35 \\
\hline \multirow{2}{*}{$\begin{array}{l}\text { essential oil } \\
\text { of mastic gum }\end{array}$} & 10 & - & - & - & - \\
\hline & 100 & - & 11 & 10 & - \\
\hline
\end{tabular}

${ }^{a}$ Antibacterial activity has not been observed at the dose of $1 \mu \mathrm{g} / \mathrm{disk}$, and data were deleted

b \#09-87: CAM-sensitive, MNZ-resistant strain; \#09-224: CAM-resistant, MNZ-sensitive strain; \#09-243: CAM-resistant, MNZ-resistant strain; \#09-292: CAM-sensitive, MNZ-sensitive strain

i.d. $0.25 \mathrm{~mm}$, GL Sciences, Tokyo Japan) with helium as carrier gas at $1.4 \mathrm{~mL} / \mathrm{min}$ flow rate. Column temperature was initially kept for $2 \mathrm{~min}$. at $60{ }^{\circ} \mathrm{C}$, gradually increased to $180{ }^{\circ} \mathrm{C}$ at a rate of $3.5^{\circ} \mathrm{C} / \mathrm{min}$ then increased to $280{ }^{\circ} \mathrm{C}$ at a rate of $10^{\circ} \mathrm{C} / \mathrm{min}$ and kept for $5 \mathrm{~min}$. The injector and interface were set to 300 and $280{ }^{\circ} \mathrm{C}$, respectively, The gas chromatograph operated in the split mode with a split ratio of $94: 1$. The mass spectrum was monitored starting at $m / z, 40$ and ending at $m / z$ 500, with a scan 
Fig. 2 Antibacterial activity of (E)-methyl isoeugenol and $\alpha$ terpineol against \#09-292 $\mathrm{H}$. pylori clinical strain. a control, b $100 \mu \mathrm{g} /$ disk of $(E)$-methyl isoeugenol
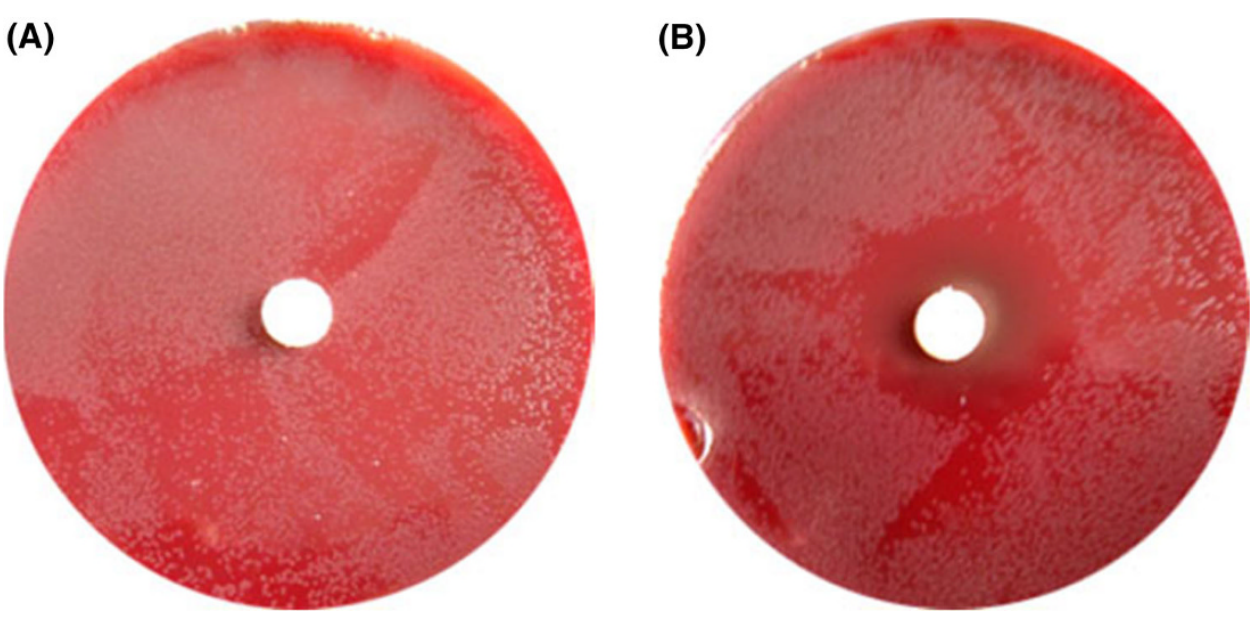

interval of $0.5 \mathrm{~s}$. and threshold of 1000 , and the solvent cut was set to $2 \mathrm{~min}$. The injection volume was $1 \mu \mathrm{L}$. The injection solution was essential oil in acetone $(50 \% \mathrm{v} / \mathrm{v})$. The chemical composition of the essential oil was analyzed using NIST and Wiley registry of mass spectral data (Shimadzu Corporation).

\subsection{H. pylori Clinical Strains}

In this study, we used four clinical isolates of $H$. pylori which were obtained from patients who underwent endoscopic examination at Oita University Hospital, Oita, Japan. Based on the Epsilometer test ( $E$ test) for drug susceptibility, these strains were assessed as sensitive or resistant to clarithromycin (CAM) and metronidazole (MNZ) at minimum inhibitory concentration (MIC) of $1 \mu \mathrm{g} / \mathrm{mL}(\mathrm{CAM})$ and $16 \mu \mathrm{g} / \mathrm{mL}$ or higher (MNZ), respectively. The clinical background and drug sensitivity of the 4 strains were as followed. CAM-sensitive, MNZ-resistant strain \#09-87 derived from atrophic gastritis; CAM-resistant, MNZ-sensitive strain \#09-224 from gastric ulcer; CAM-resistant, MNZ-resistant strain \#09-243 from atrophic gastritis; CAM-sensitive, MNZ-sensitive strain \#09-292 from gastric cancer.

\subsection{Antibacterial Activity Test}

H. pylori culture suspension was used to inoculate the plates, and discs containing $1,10,100 \mu \mathrm{g}$ of compounds were applied onto culture plates. The plates were incubated under microaerophilic conditions for 3 days at $37^{\circ} \mathrm{C}$ on Mueller Hinton II Agar (Becton Dickinson, Franklin Lakes, NJ, USA) plate supplemented with $7 \%$ horse blood without antibiotics. The antibacterial activities were evaluated by measuring a diameter of the inhibition ring.
Acknowledgments We thank Mr. Hideo Minobe (Alliance Co., Ltd, Fukuoka, Japan) for supply us the mastic gum and also fruitful discussion.

Conflict of interest The authors declare no conflict of interest.

Open Access This article is distributed under the terms of the Creative Commons Attribution License which permits any use, distribution, and reproduction in any medium, provided the original author(s) and the source are credited.

\section{References}

1. V. Herrera, J. Parsonnet, Clin. Microbiol. Infect. 15, 971-976 (2009)

2. L.E. Wroblewski, R.M. Peek Jr, K.T. Wilson, Clin. Microbiol. Rev. 23, 713-739 (2010)

3. S. Kabir, Helicobacter 14, 159-171 (2009)

4. K. Fukase, M. Kato, S. Kikuchi, K. Inoue, N. Uemura, S. Okamoto, S. Terao, K. Amagai, S. Hayashi, M. Asaka, Lancet 372, 392-397 (2008)

5. H. Suzuki, T. Nishizawa, T. Hibi, Future Microbiol. 5, 639-648 (2010)

6. C. Hernández-Hernández Ldel, E.C. Lazcano-Ponce, Y. LópezVidal, G.R. Aguilar-Gutiérrez, Salud. Pública. Méx 51, 447-454 (2009). Suppl 3

7. S.F.H. Zaidi, I. Yoshida, F. Butt, M.A. Yusuf, K. Usmanghani, M. Kadowaki, T. Sugiyama, Biol. Pharm. Bull. 32, 631-636 (2009)

8. F.U. Huwez, D. Thirlwell, A. Cockayne, D.A.A. Ala'Alden, New Eng. J. Med. 339, 1946 (1998)

9. C. Koutsoudaki, M. Krsek, A. Rodger, J. Agri. Food Chem. 53, 7681-7685 (2005)

10. P. Magiatis, E. Melliou, A.K. Skaltsounis, I.B. Chinou, S. Mitaku, Planta Med. 65, 749-752 (1999)

11. L. Lauk, S. Ragusa, A. Rapisadra, S. Franco, V.M. Nicolosi, J. Chemother. 8, 207-209 (1996)

12. S. Paraschos, P. Magiatis, S. Mitakou, K. Petraki, A. Kalliaropoulos, P. Maragkoudakis, A. Mentis, D. Sgouras, A.L. Skaltsounis, Antimicrob. Agents Chemother. 51, 551-559 (2007)

13. N. Uemura, S. Okamoto, S. Yamamoto, N. Matsumura, S. Yamaguchi, M. Yamakido, K. Taniyama, N. Sasaki, R.J. Schlemper, New Eng. J. Med. 345, 784-789 (2001) 
14. T. Ohkusa, I. Takashimizu, K. Fujiki, S. Suzuki, K. Shimoi, T. Horiuchi, T. Sakurazawa, K. Ariake, K. Ishii, J. Kumagai, T. Tanizawa, Ann. Intern. Med. 129, 712-715 (1998)

15. M. Stolte, S. Eidt, Lancet 342, 568 (1993)

16. A.C. Wotherspoon, C. Doglioni, T.C. Diss, L. Pan, A. Moschini, M. de Boni, P.G. Isaacson, Lancet 342, 575-577 (1993)

17. E.M. El-Omar, I.D. Penman, J.E.S. Ardill, R.S. Chittajallu, C. Howie, K.E.L. McColl, Gastroenterology 109, 681-691 (1995)
18. Therapeutic guideline for Helicobacter pylori infection 2009. Jpn. J. Helicobacter Res. 10 suppl, 1-25. http://www.jshr.jp/. Accessed 15 March 2009

19. E. Rimbara, N. Noguchi, M. Tanabe, T. Kawai, Y. Matsumoto, S.M. Asatsu, Clin. Microbiol. Infect. 11, 307-311 (2005) 